\title{
Characterization of $\mathrm{La}_{1-x} \mathrm{Sr}_{1+x} \mathrm{Cu}_{5-x} \mathrm{Fe}_{x} \mathrm{O}_{12+\delta}(0 \leqslant x \leqslant 1 \cdot 0)$ by dc electrical conductivity, magnetic susceptibility and EPR measurements
}

\author{
B B DAS*\#: C MICHEL and B RAVEAU \\ Laboratoire de Crystallographie et sciences des Matériaux, (CRISMAT). ISMRA, Boulevard \\ du Maréchal Iuin, 14050 Caen, Cedex, France \\ \#Present Address: Department of Chemistry, Pondicherry University, Pondicherry 605014. \\ India \\ MS received 15 April 1996; revised 27 August 1996

\begin{abstract}
The DC electrical resistivity results of $\mathrm{La}_{+}{ }_{x} \mathrm{Sr}_{1+x} \mathrm{Cu}_{5}{ }_{x} \mathrm{Fe}_{x} \mathrm{O}_{12+\lambda}(0 \leqslant x \leqslant 1 \cdot 0)$ showed that for $S 1(x=0)$ and $S 2(x=0.25)$ the temperature coefficient of resistivity (TCR). $\mathrm{d} \rho / \mathrm{d} T$, is positive and slightly increases with increasing temperature in the range $20.270 \mathrm{~K}$. This shows the metallic nature of $S 1$ and $S 2$. For the samples $S 3(x=0.5)$ and $S 4(x=0.75)$. TCR slightly increases in the range $20-270 \mathrm{~K}$, with change in sign from negative to positive at $\sim 80 \mathrm{~K}$ and $\sim 130 \mathrm{~K}$, respectively. These results show the metal-insulator type transition in S3 and $S 4$. For the sample $S 5(x=1 \cdot 0)$, the TCR is negative and gradually increases in the range $20-270 \mathrm{~K}$, which shows its semiconductor-like behaviour. The activation energy for S.5 is found to be $021 \times 10^{2} \mathrm{eV}$. Furthermore. the DC resistivity results of $\mathrm{S} 1 . \mathrm{S} 5$ in the range $350-660 \mathrm{~K}$ are in conformity with the low temperature results. The very weak temperature dependence of magnetic susceptibility results of $\mathrm{S} 1$ - S3 show Palu-paramagnetic behaviour in the range $77 \mathrm{~K}-400 \mathrm{~K}$, while $\mathrm{S} 4$ and $\mathrm{S} 5$ exhibit Pauli-paramagnetic behaviour in the range $77-850 \mathrm{~K}$. Long-range antiferromagnetic interaction is observed in $\mathrm{S} 5(x=1.0)$ below $T_{c} \sim 100 \mathrm{~K}$. The room temperature EPR lineshapes gradually improve from metallic $\left.\mathrm{S} / \mathrm{x}=0\right)$ increasing trend in $g_{\text {iso }}$-values of 1.880 in $\$ 2$ to 1.961 in 55 . However. the $q_{\text {iso }}$-value for $S 1$ could not be ohserved due to very poor lineshape.
\end{abstract} \\ to semiconductor-like $\mathrm{S} 5(x=1.0)$. Negative $f-s h i f t$ is observed in the samples S2 $-\mathrm{S} 5$ with
}

Keywords. Electron paramagnetic resonance: magnetic suscentibility; do electrical resistivity; cuprates.

\section{Introduction}

The oxygen deficient perovskites (Michel et al 1985, 1987) can exist as superconductors (Bednorz and Müller 1985; Rao et al 1987; Torrance et ai 1988). as metallic nonsuperconducting compounds like $\left\{\mathrm{La}_{1} \ldots \mathrm{Sr}_{x}\right\}_{8} \mathrm{Cu}_{8} \mathrm{O}_{20}(0 \cdot 16 \leqslant x \leqslant 0 \cdot 24)$ (Er-Rakho et al 1988). ( $\left.\mathrm{La}_{1-x} \mathrm{Sr}_{x}\right)_{8} \mathrm{Cu}_{8} \mathrm{O}_{2 n-x}(x=0 \cdot 1666,0 \cdot 2,0 \cdot 2381$. 0.25) (Otzschi et al 1992). $\left(\mathrm{La}_{1-x} \mathrm{Sr}_{x}\right)_{8} \mathrm{Cu}_{4} \mathrm{O}_{16}(x=0 \cdot 1429)$ (Otzschi and Ueda 1992) and as semiconductor-like as in $\left(\mathrm{La}_{1-x} \mathrm{Sr}_{x}\right)_{8} \mathrm{Cu}_{8} \mathrm{O}_{16+\delta}(x=0.70-0 \cdot 80)$ (Otzschi et al 1993) depending on their composition and structures. Considering the wide application potentialities of these perovskites as superconducting to semiconducting materials, it is worth investigating these materials to understand their structure and physical properties. In this paper, we report our investigations on characterizing $\mathrm{La}_{4-x} \mathrm{Sr}_{1+x} \mathrm{Cu}_{5-x} \mathrm{Fe}_{x} \mathrm{O}_{12+\delta}(0 \leqslant x \leqslant 10)$ by $\mathrm{DC}$ electrical conductivity measurements, magnetic susceptibility measurements and electron paramagnetic resonance (EPR) spectroscopy.

*To whom all correspondence should he addiressed.

a EEC Postdoctoral Fellow 


\section{Experimental}

The samples were prepared by solid state reaction. The appropriate mixtures of dried $\mathrm{La}_{2} \mathrm{O}_{3}, \mathrm{CuO}$ and $\mathrm{Fe}_{2} \mathrm{O}_{3}$ were heated in platinum crucibles at $1173 \mathrm{~K}$ for $6 \mathrm{~h}$ followed by heating the samples at $1273 \mathrm{~K}$ for $24 \mathrm{~h}$, in air atmosphere. The compositions of the samples are shown in table 1.

The bars from the powdered samples were prepared at 104 daN pressure by a hydraulic press and sintered at $1273 \mathrm{~K}$ for $18 \mathrm{~h}$. The bars were then polished on emery papers and accurate dimensions were taken. The $\mathrm{DC}$ electrical resistivity measurements of the bars were carried out by four-probe method in the range $20-270 \mathrm{~K}$ at $1 \mathrm{~K}$ interval in air atmosphere and in the range $350-660 \mathrm{~K}$ at $5 \mathrm{~K}$ interval in $250 \mathrm{mbar} \mathrm{He}$ atmosphere. The magnetic susceptibility of the samples were measured using a Setaram Faraday balance in an external magnetic field of $0.3 \mathrm{~T}$ in the temperature range $77-850 \mathrm{~K}$ and further for the sample $S 5$ in the range $4 \cdot 2-300 \mathrm{~K}$. Finely powdered samples were used to record the EPR spectra of the samples. The X-band EPR spectra at $300 \mathrm{~K}$ were recorded on a Bruker spectrometer system (Model ER 200D) fitted with a double cavity $\mathrm{TE}_{110}$. In all the cases, $100 \mathrm{kHz}$ magnetic field modulation was used. The measured $g$-values were calibrated with respect to the Bruker strong pitch ( $g=2.0028)$.

\section{Results and discussion}

In the above series, $\mathrm{La}_{4-x} \mathrm{Sr}_{1+x} \mathrm{Cu}_{5-x} \mathrm{Fe}_{x} \mathrm{O}_{12+\delta}$, the samples were prepared in the range $0 \leqslant x \leqslant 3.5$ using the preparation procedures mentioned above. Interestingly samples in the range $0 \leqslant x \leqslant 1.0$ form tetragonal phase, whereas in the range $1 \cdot 2 \leqslant x \leqslant 2 \cdot 0$, mixed tetragonal and cubic phases and in the range $2 \cdot 5 \leqslant x \leqslant 3 \cdot 5$, the cubic phase are formed. In this paper, our study is restricted to the samples in the tetragonal phase in the range $0 \leqslant x \leqslant 1 \cdot 0$. The oxygen contents in the samples $\mathrm{S} 1-\mathrm{S} 5$ were determined by DTA and TG analyses as discussed elsewhere (Das 1993).

In figure 1a, we present our observed DC electrical resistivity versus temperature plots in the range $20-270 \mathrm{~K}$ of samples S1-S5 (table 1). The plots show that in the samples $S 1(x=0)$ and $S 2(x=0 \cdot 25)$, the temperature coefficient of resistivity (TCR), $\mathrm{d} \rho / \mathrm{d} T$ is positive and slightly increases with increasing temperature in the range $20-270 \mathrm{~K}$. This behaviour can be characterized by the following equation for the specific conductivity $\sigma$, of metals or metallic conductors (West 1986a)

$$
\sigma=\sum_{\mathrm{i}} n_{\mathrm{i}} e_{\mathrm{i}} \mu_{\mathrm{i}}
$$

Table 1. Compositions of the samples studied in the series $\mathrm{La}_{4-x} \mathrm{Sr}_{1+x} \mathrm{Cu}_{5-x} \mathrm{Fe}_{x} \mathrm{O}_{12+3}(0 \leqslant x \leqslant 1 \cdot 0)$.

\begin{tabular}{lll}
\hline Sample & \multicolumn{1}{c}{ Compositional formula } & $x$ \\
\hline $\mathrm{S} 1$ & $\mathrm{La}_{4} \mathrm{SrCu}_{5} \mathrm{O}_{12+0.43}$ & 0 \\
$\mathrm{~S} 2$ & $\mathrm{La}_{3.75} \mathrm{Sr}_{1 \cdot 25} \mathrm{Cu}_{4.75} \mathrm{Fe}_{0.25} \mathrm{O}_{12+0.24}$ & 0.25 \\
$\mathrm{~S} 3$ & $\mathrm{La}_{3.5} \mathrm{Sr}_{1.5} \mathrm{Cu}_{4.5} \mathrm{Fe}_{0.5} \mathrm{O}_{12+0.21}$ & 0.5 \\
$\mathrm{~S} 4$ & $\mathrm{La}_{3 \cdot 25} \mathrm{Sr}_{1 \cdot 75} \mathrm{Cu}_{4 \cdot 25} \mathrm{Fe}_{0.75} \mathrm{O}_{12+0.17}$ & 0.75 \\
$\mathrm{~S} 5$ & $\mathrm{La}_{3} \mathrm{Sr}_{2} \mathrm{Cu}_{4} \mathrm{FeO}_{12+0.10}$ & 1.0 \\
\hline
\end{tabular}



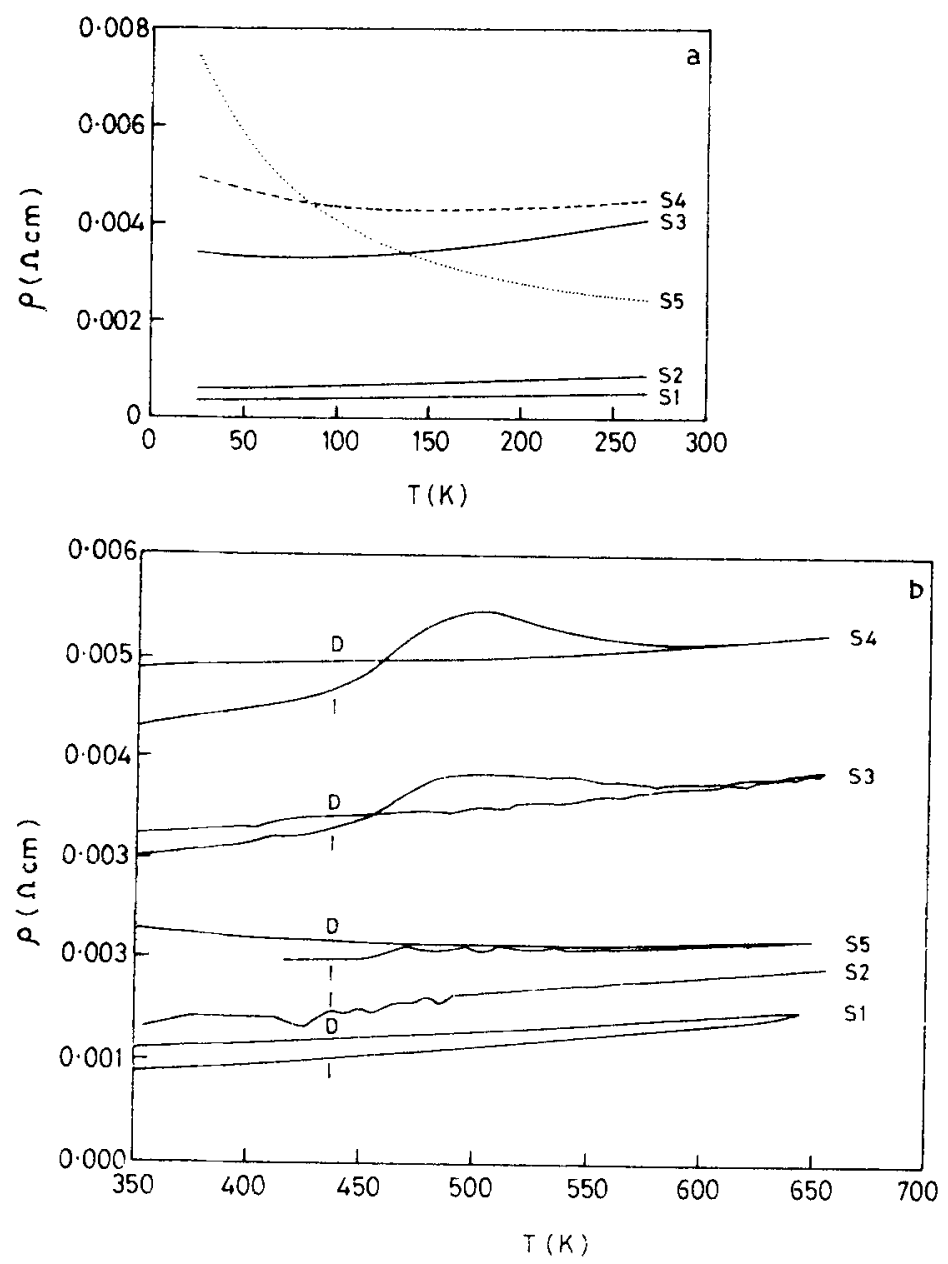

Figure 1. a. DC electrical resistivity, $\rho(\Omega \mathrm{cm})$ vs $T(\mathrm{~K})$ in the temperature range of $20-270 \mathrm{~K}$ of samples $S 1(x=0)$. S2 $(x=0 \cdot 25), S 3(x=0.5), S 4(x=0 \cdot 75)$ and $S 5(x=1 \cdot 0)$ (table 1) and b. DC electrical resistivity, $\rho(\Omega \mathrm{cm})$ vs $T(\mathrm{~K})$ in the temperature range of $350-660 \mathrm{~K}$ of samples $S 1(x=0), S 2(x=0.25), S 3(x=0.50), S 4(x=0.75)$ and $S 5(x=1 \cdot 0)$ (table 1). I indicates increasing and $D$ indicates decreasing temperatures.

where $\sigma=1 / \rho$ and $n_{\mathrm{i}}$ is the charge carrier concentration with charge $e_{\mathrm{i}}$ and mobility $\mu_{\mathrm{i}}$. The above results show that $\mathrm{S} 1$ and $\mathrm{S} 2$ exhibit metallic behaviour. In the cases of the samples $S 3(x=0.5)$ and $S 4(x=0.75)$, the plots (figure 1a) show that at low temperature the TCR $(\mathrm{d} \rho / \mathrm{d} T)$ is negative and increases in magnitude with increasing temperature until the change in sign of the TCR occurs. Above the transition temperature, in each case of S3 and S4, the TCR is positive and slightly increases in magnitude up to $270 \mathrm{~K}$. The change in sign in TCR $(\mathrm{d} \rho / \mathrm{d} T)$ through minima is $\sim 80 \mathrm{~K}$ for $\mathrm{S} 3$ and $\sim 130 \mathrm{~K}$ for $\mathrm{S} 4$. Such behaviour is well known in metallic alloy and metallic glasses containing d-elements, but is rather rare in metallic (or insulating) oxides or perovskites. Four main effects can account for such a minimum in the $\rho$ versus temperature plots as pointed out by Howson and Gallaghar (1988). The first one is the well known Kondo effect (Ashcroft and Mermin 1976). We think that if some magnetic $\mathrm{Cu}^{3+}$ or 
$\mathrm{Cu}^{2+}$ and/or $\mathrm{Fe}^{4+}$ or $\mathrm{Fe}^{3+}$ ions were responsible for Kondo effect, it would be at least present in the samples $\mathrm{S} 1+\mathrm{S} 4$. since $\mathrm{S} 1$ contains mixed valence $\mathrm{Cu}^{3+}$ and $\mathrm{Cu}^{2+}$ as discussed by Er-Rakho et al (1988). Also our Mössbauer study (Das 1993) of a Fe containing sample with $x=3 \cdot 0$, having cubic phase, revealed that $72 \% \mathrm{Fe}^{3+}$ and $28 \%$ $\mathrm{Fe}^{4+}$ ions are formed in the lattice. The other effects are: scattering from two-level states but it seems only applicable to glasses and amorphous materials (Howson and Gallaghar 1988), and quantum interference effect. Elastic collision with small relaxation times may give rise to quantum interferences which increase the resistivity at low temperature where they are expected to be the main scattering mechanism (Mott 1991). Quantum interferences are destroyed by inelastic scattering so that, as $T$ increases phonon scattering leads to decreased resistivity. However, at higher temperatures normal inelastic phonon scattering becomes the predominant mechanism and the usual increase of $\rho$ versus temperature is observed. The fourth effect which can give rise to a decrease of $\rho$ with increasing temperature in a metallic system results from long-range electron-electron interactions (Altschuler and Aronov 1979). Such longrange interaction appears to be feasible in the systems studied. Er-Rakho et al (1988) reported the structure of the Fe free compound $S 1(x=0)$. The structure is built of corner-sharing $\mathrm{CuO}_{6}$ octahedra, $\mathrm{CuO}_{5}$ pyramids and $\mathrm{CuO}_{4}$ square planar groups forming hexagonal tunnels in which are located the $\mathrm{La}^{3+}$ and $\mathrm{Sr}^{2+}$ ions. Furthermore, we discussed elsewhere (Das 1993) from the Rietveld analysis of the X-ray powder diffraction results of the Fe incorporated sample $\mathrm{S} 5(x=1 \cdot 0)$ that $\mathrm{Fe}$ and $\mathrm{Cu}$ are statistically distributed in the $\mathrm{MO}_{6}(\mathrm{M}=\mathrm{Cu}$ or $\mathrm{Fe})$ octahedra. Thus long-range interaction of electrons occurs through superexchange (West 1986b) of electrons between $\mathrm{Cu}$ or $\mathrm{Fe} 3 \mathrm{~d}$ orbitals via corner-shared p-orbitals of oxygen. The above third and fourth effects are expected to occur near the metal-insulator transition and lead to electronic localization (Howson and Gallagher 1988; Mott 1991). Another origin for a tendency to localization even applicable in an impurity-free or defect free crystal is the possibility of a strong electron-phonon interaction which can eventually lead to formation of polaron (Rao and Gopalakrishnan 1986).

Again, for the sample S5 $(x=1 \cdot 0)$ (figure 1 a) the temperature coefficient of resistivity $(\mathrm{d} \rho / \mathrm{d} T)$ is negative within the temperature range of $20-270 \mathrm{~K}$ and gradually increases in magnitude from $20 \mathrm{~K}$ to $270 \mathrm{~K}$. This result shows the semiconductor-like behaviour in $S 5(x=1.0)$ in the range 20 to $270 \mathrm{~K}$. Using the Arrhenius relation as under

$$
\rho=\rho_{0}^{E / k T}
$$

we calculate the activation energy, $E$, to be $0.2 \times 10^{-2} \mathrm{eV}$. As expected the sample S5 exhibiting semiconductor-like behaviour has extremely low DC activation energy.

From figure 1a, it is also interesting to note that the resistivities of the samples increase gradually from $S 1(x=0)$ to $S 4(x=0.75)$ with increasing Fe content at all temperatures in the range $20-270 \mathrm{~K}$. This result shows that the presence of $\mathrm{Fe}$ ions in the matrix increases the resistivities of the samples. Furthermore, the increasing trend in resistivity depends on the concentration of the Fe ions present in the matrix.

In figure $1 b$, we report our observed DC electrical resistivity, $\rho$ versus temperature plots in the range $250-650 \mathrm{~K}$, of the samples S1-S5 (table 1). In the plots, I indicates resistivity with increasing temperature and $D$ indicates resistivity with decreasing temperature. As in the cases for the low temperature resistivity behaviour in the range $20-270 \mathrm{~K}$, in the high temperature range $350-360 \mathrm{~K}$ also, the TCR, $(\mathrm{d} \rho / \mathrm{d} T)$, is positive 
in the I and D cases for the samples S3 and S4. Furthermore, the value of TCR $(\mathrm{d} \rho / \mathrm{d} T)$ gradually increases from $20 \mathrm{~K}$ to $270 \mathrm{~K}$, in the cases of increasing, I and decreasing, $\mathrm{D}$ temperatures for S1--S4 (table 1) in the above particular cases. This is in agreement with our low temperature results as discussed earlier. However, in the cases of samples $\mathrm{S} 3$ and $\mathrm{S} 4$, the resistivity behaviour with increasing, I, temperature is rather complex. In these cases, the TCR, $(\mathrm{d} \rho / \mathrm{d} T)$ changes sign from positive to negative, $\sim 500 \mathrm{~K}$, and then again at $\sim 660 \mathrm{~K}$ from negative to positive. It is interesting to mention here that for the samples S3 $(x=0.5)$ and $S 4(x=0.75)$ in the low temperature case (figure 1a), the resistivities are measured with increasing temperature. In these cases also, change in the sign of TCR $(\mathrm{d} \rho / \mathrm{d} T)$ from negative to positive occur at $\sim 80 \mathrm{~K}$ for $\mathrm{S} 3$ and $\sim 130 \mathrm{~K}$ for $\mathrm{S} 4$. This shows the complex resistivity behaviour of S3 and S4 with increasing temperature in both the temperature ranges studied. However, in the cases of decreasing temperature for S3 and S4, the TCR does not change sign in both the cases, and from the difference in the $\rho$ versus $T$ plots for increasing and decreasing temperature cases, we infer that for increasing temperature for S3 and S4 (figures $1 \mathrm{a}$ and b) the temperatures at which TCR changes sign, an irreversible phase transition occurs. In the case of the sample $\mathrm{S} 5(x=1.0)$ (figure $1 \mathrm{~b})$, the TCR $(\mathrm{d} \rho / \mathrm{d} T)$, is negative and increases in magnitude slightly from $350 \mathrm{~K}$ to $650 \mathrm{~K}$, for both increasing $\mathrm{l}$, and decreasing $\mathrm{D}$, cases. This result shows the semiconductor-like behaviour of the sample in the temperature range $350 \mathrm{~K}-660 \mathrm{~K}$, and is in agreement with low temperature result of $S 5(x=1 \cdot 0)$ in the range $20-270 \mathrm{~K}$, as discussed earlier.

The thermal variation of $1 / \chi$, the inverse magnetic susceptibility of the samples are shown in figure $2 \mathrm{a}$. In the cases of samples $S 4(x=0.75)$ and $S 5(x=1.0)$ the experimental points were fitted with the usual Curie- Weiss law,

$$
\chi_{\mathrm{M}}=\frac{C_{\mathrm{M}}}{T-\theta}+T I P
$$

and the values of the Curie constant, $C_{M}$, and TIP parameters are shown in table 2 along with the $g_{\text {iso }}$-values of the samples S1-S5 (table 1). The $g_{\text {iso }}$-values of the samples will be discussed later. The table shows that values of the Curie constant, $C_{M}$ are fairly low. However, these values could not be ascribed to a particular metal ion, since the samples $\mathrm{S} 4$ and $\mathrm{S} 5$ contain mixed valence $\mathrm{Cu}$ and $\mathrm{Fe}$ ions.

The observed TIP values are much larger than the Van Vleck term, which may be approximately estimated as $70-80 \times 10^{-6} \mathrm{emu} \mathrm{mol}^{-1}$ (Figgis 1961). The larger part of this TIP value we ascribe due to the Pauli paramagnetic behaviour of the compounds. Pauli type paramagnetism in such cuprates is also discussed by Otzschi and Ueda (1992) and Otzschi et al (1992, 1993).

In samples S1-S3 (figure $2 \mathrm{a}$ ) the temperature variations of inverse susceptibility, $1 / \chi$, show rather complex behaviour. From $77 \mathrm{~K}$ to $\sim 400 \mathrm{~K}$ the weak temperature dependence of the magnetic susceptibility of the samples S1-S3 shows the Pauli paramagnetic behaviour in the above temperature range. However, above $\sim 400 \mathrm{~K}$ in the above samples steep increase in $1 / \chi$ value is observed. It is interesting to note that this steep increase in $1 / \chi$ values have decreasing trend from $S 1(x=0)$ to $S 3(x=0 \cdot 50)$ through $S 2$ $(x=0 \cdot 25)$.

Figure $2 b$ shows the inverse susceptibility, $1 / \mathcal{x}$, versus $T(K)$ plot of $S 5(x=1 \cdot 0)$ (table 1) in the temperature range $4 \cdot 2-300 \mathrm{~K}$. The weak temperature dependence of the magnetic susceptibility shows the Pauli paramagnetic behaviour of S5 $(x=1.0)$ in the 


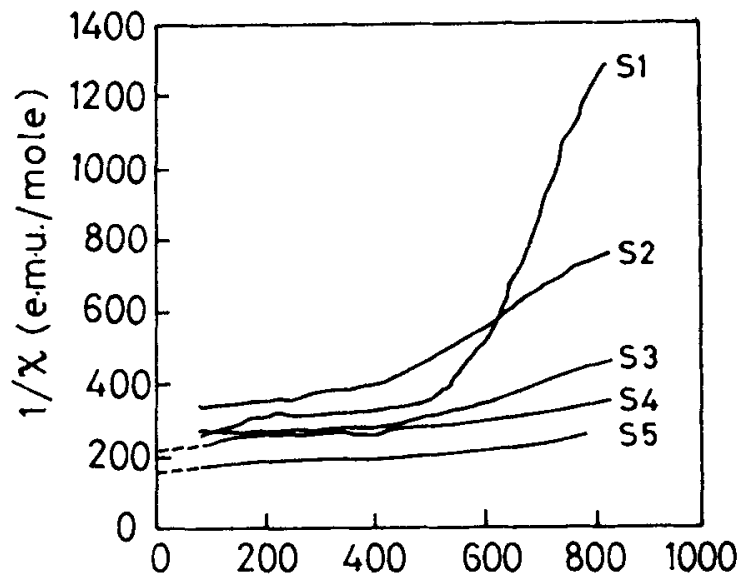

TEMPERATURE, T $(K)$ a

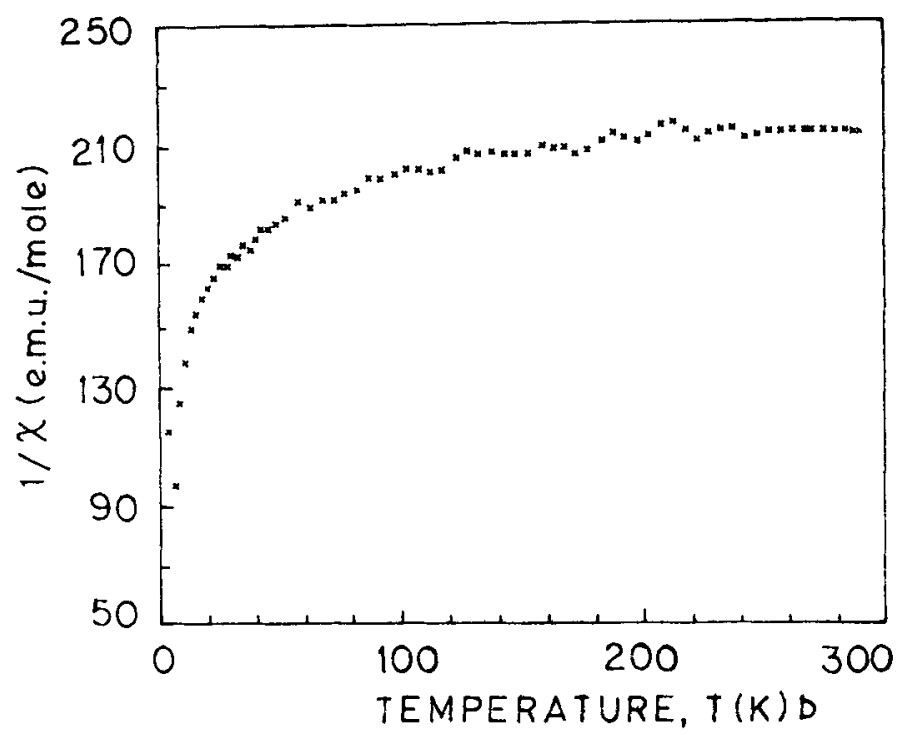

Figure 2. Inverse susceptibility, $1 / \chi$, vs $T(\mathrm{~K})$ in the temperature range of $77-850 \mathrm{~K}$ of samples $\mathrm{S} 1(x=0), \mathrm{S} 2(x=0.25), \mathrm{S} 3(x=0.50), \mathrm{S} 4(x=0.75), \mathrm{S} 5(x=1.0)$ (table 1$)$ and $\mathrm{b}$. inverse susceptibility, $1 / \chi$, vs $T(\mathrm{~K})$ in the temperature range of $4 \cdot 2-300 \mathrm{~K}$ of $\mathrm{La}_{3} \mathrm{Sr}_{2} \mathrm{Cu}_{4} \mathrm{FeO}_{12+0.1}$ $(x=1 \cdot 0)($ table 1$)$.

temperature range $\sim 100-300 \mathrm{~K}$ as discussed above in figure $2 \mathrm{a}$ also. However, the sharp decrease in $1 / \chi$ value below $100 \mathrm{~K}$ up to $4.2 \mathrm{~K}$ shows the long range antiferromagnetic coupling at lower temperature with Curie temperature $T_{\mathrm{c}} \sim 100 \mathrm{~K}$.

Our results on EPR studies will be discussed now. In figure 3 we present our observed EPR spectra of samples $S 1(x=0)$ to $S 5(x=1 \cdot 0)$ at room temperature. We know from our DC electrical resistivity results as discussed earlier that $S 1$ and $S 2$ are metallic; S3 and S4 exhibit transition from semiconductor-like to metallic at $\sim 80 \mathrm{~K}$ and $\sim 130 \mathrm{~K}$, respectively, whereas $\mathrm{S} 5$ is semiconductor-like. This behaviour is reflected in the nature of the gradually improved EPR lineshapes of the samples from S1 $(x=0)$ to $\mathrm{S} 5(x=1 \cdot 0)$. The extremely weak lineshape in $\mathrm{S} 1(x=0)$ shows the presence of 
Table 2. Curie constant, $C_{M}$, and TIP of samples $S 4(x=0.75)$ and $S 5(x=1.0)$ and $g_{\text {iso }}$-values of the samples (table 1) in the series $\mathrm{La}_{4-x} \mathrm{Sr}_{1+x} \mathrm{Cu}_{5}{ }_{x} \mathrm{Fe}_{x} \mathrm{O}_{12+\delta}(0 \leqslant x \leqslant 1 \cdot 0)$.

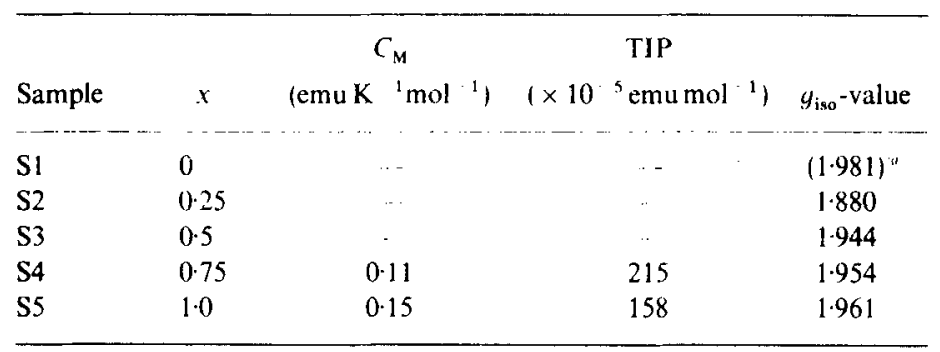

"unomalous $g_{\text {iso }}$-values of 1.981 for $\mathrm{S} 1$ is discussed in the text.

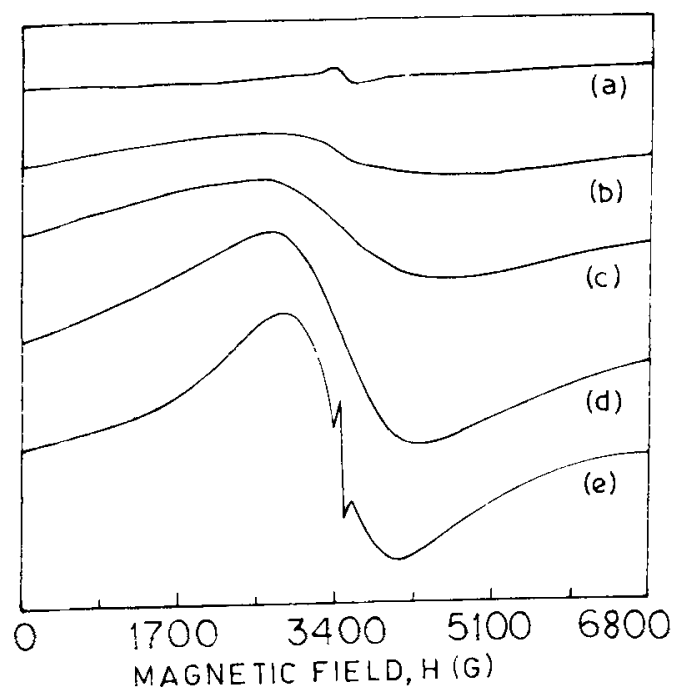

Figure 3. EPR spectra of the samples in the series $\mathrm{La}_{4-x} \mathrm{Sr}_{1+x} \mathrm{Cu}_{5-x} \mathrm{Fe}_{x} \mathrm{O}_{12+\delta}$ recorded at room temperature: (a) $S 1(x=0)$, (b) $S 2(x=0.25)$, (c) $S 3(x=0.5)$, (d) $S 4(x=0.75)$ and (e) S5 $(x=1 \cdot 0)($ table 1$)$.

highly delocalized electrons in the lattice due to its metallic behaviour, whereas in S5 $(x=1 \cdot 0)$, due to its semiconductor-like behaviour the electrons are less delocalized in the lattice and as a result, comparatively improved EPR lineshape is observed.

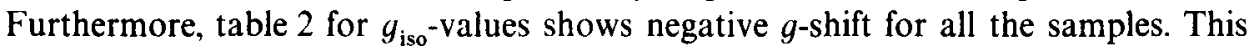
shows positive nature of the spin-orbit coupling constant. However, such values cannot be ascribed to any particular paramagnetic ion, since all the samples contain more than one paramagnetic ions.

Furthermore, it is known from literature (Er-Rakho et al 1988) that the sample S1 $(x=0)$ has mixed valence of $\mathrm{Cu}^{2+}$ and $\mathrm{Cu}^{3+}$ ions. Both the $\mathrm{Cu}^{2+}$ and $\mathrm{Cu}^{3+}$ ions have negative values of spin-orbit coupling constant (Abragam and Bleaney 1970). Thus a $g_{\text {iso }}$-value of 1.981 with negative $g$-shift is an anomalous nature observed for S1. It appears plausible that because of the metallic nature of S1 a very broad and undetectable EPR signal (Ziman 1964) results and the very weak signal at the middle could be 
due to impurity present in the sample. However, with the gradual increase in $g_{\text {iso }}$-value of 1.880 in $S 2(x=0.25)$ to 1.961 in $S .5(x=1.0)$, we ascribe to the gradual change in the net crystal field effect on $\mathrm{Cu}$ and $\mathrm{Fe}$ ions present in the matrices.

\section{Conclusion}

From our above studies the DC electrical resistivity results in the range $20-270 \mathrm{~K}$ show that $S 1(x=0)$ and $S 2(x=0.25)$ exhibit metallic behaviour, while $S 3(x=0.50)$ and $S 4$ $(x=0.75)$ exhibit metal-insulator type transition at $\sim 80 \mathrm{~K}$ and $\sim 130 \mathrm{~K}$, respectively. The sample S5 $(x=1.0)$ exhibits semiconductor-like behaviour in the range $20-270 \mathrm{~K}$. Furthermore, the DC resistivity results in the range $350-650 \mathrm{~K}$ are also in conformity with the low temperature results. The magnetic susceptibility results show that $\mathrm{S} 1-\mathrm{S} 3$, show Pauli-paramagnetic behaviour in the range $77 \mathrm{~K}$ to $\sim 400 \mathrm{~K}$, while S4 and S5 exhibit Pauli-paramagnetic behaviour in the range $77-850 \mathrm{~K}$. In the case of $\mathrm{S} 5$ $(x=1 \cdot 0)$, long-range antiferromagnetic interaction is observed at lower temperature with $T_{\mathrm{c}} \sim 100 \mathrm{~K}$. The EPR lineshapes improve gradually in the samples from metallic $(x=0)$ to semiconductor-like $(x=1 \cdot 0)$. Negative $g$-shift is observed in the case of the samples $\mathrm{S} 2-\mathrm{S} 5$. However, for $\mathrm{S} 1$, the $g_{\mathrm{iss}}$-value could not be observed due to very poor lineshape.

\section{Keferences}

Abragan A and Bleaney B 1970 Electron paranagnetic resonance of transition ions (Oxford: Clarendon Press) p. 399

Altschuler B L and Aronov A G 1979 Solid State Commun. 30115

Ashcroft N W and Mermin N D 1976 Solid slate physics (New York: Hait, Rinehart, and Winston) Bednorz J G and Müller K A 1985 2. Phys. B4 294

Das B B 1993 Synthesis and characterization of $\mathrm{La}_{4-x} \mathrm{Sr}_{1+x} \mathrm{Cu}_{5-x} \mathrm{Fe}_{x} \mathrm{O}_{12+\delta}(0 \leqslant x \leqslant 3.5)$ perouskites, Post Doctoral Report of the EC, Brussel, Belgium

Er-Rakho L, Michel C and Raveau B 1988 J. Solid State Chem. 73514

Figgis B N 1961 Introduction to ligand fields (New York: Inter science)

Howson M A and Gallagher B L 1988 Phys. Rep. 170265

Michel C, Er-Rakho L and Raveau B 1985 Mater. Res. Bull. 20667

Michel C, Er-Rakho L, Hervieu M. Pannetier J and Raveau B 1987 J. Solid State Chem. 68143

Mott N F 199! Metal-insulator transition (London: Taylor and Francis) 2nd ed

Otzschi K and Ueda Y 1992 Technical Report, ISSP Tokyo, Japan, Ser. A No. 2612

Otzschi K, Hayashi A, Fujiwara Y and Ueda Y 1992 Technical Report, iSSP Tokyo, Japan Ser. A No. 2583

Otzschi K, Koga K and Ueda Y 1993 Technical Report, ISSP Tokyo, Japan, Ser. A No. 2669

Rao C N R and Gopalakrishnan J 1986 New directions in solid state chemistry (Cambridge: Cambridge University Press) p. $26^{\circ}$

Rao C N R, Ganguly P. Shreedha: K, Mohan Ram R A and Sarode P A 1987 Mater. Res. Bull. 22849

Torrance J B, Tokura Y, Nazzal A and Parkin S S 1988 Phys. Ret. Lett. 60542

West A R 1986a Solid state chemistiy and its applications (New York: John Wiley and Sons) p. 453

West A R 1986b Solid state chemistry and its applications (New York: John Wiley and Sons) p. 560

Ziman Z M 1964 Principles of the theory of solids (Cambridge: Cambridge University Press) 